\title{
The unmatched Public Health Humanitarian Emergencies Context with persistently outdated methods of response -Case of South Sudan 2017
}

\author{
Article by Sebit Musfafa \\ PhD in Public Health, Texila American University, South Sudan \\ E-mail: Sebison81@gmail.com
}

\begin{abstract}
Introduction: This study aims to determine the unmatched Public Health Humanitarian Emergencies Context with recurrently outdated methods of response -South Sudan is used as a case study. With specific objective: to increases the support for vital, approach in Public health activities in humanitarian emergencies and ascertain new ways of delivering Public health activities in humanitarian emergencies system.

Methodology: The study, was secondary research to track down useful existing published information and a review of the secondary literature on Public health activities in humanitarian emergencies, the principle investigator summaries the articles from South Sudan NGOs forum, BioMed central, Web of Science (2005-2017) and PubMed (2005-2017).

Results: South Sudan reported the highest Humanitarian access incidence in the region, most frequent report in 2017, (101) in August 2017 laterally, and most implicate violence against humanitarian workers and properties. Evidence indicate South Sudan is not receiving enough support and appropriate global focused, due jeopardy, that lead to losing more humanitarian support. Yet, it's not the humanitarian agencies that's going to solve the Public health humanitarian emergencies in South Sudan, than the political will and support.

Conclusion: South Sudan situation cannot be put in to one box, because it has many vigorous, therefore need different approaches and interventions. The study recommends that improved data are imperative towards supporting inter-sectorial work in addressing Public health care needs of the persons of concern to Humanitarian agencies.
\end{abstract}

Keywords: Complex emergency, humanitarian, Health Information System (HIS) - Public health -0 persons of concern to Humanitarian agencies.

\section{Introduction}

The South Sudanese crisis has become the largest and most complex emergency in Africa. Security in South Sudan continues to deteriorate and close to four million people - one-third of the total populationare displaced. More than 1.7 million South Sudanese have fled the country and an estimated 7.5 million people are in need of urgent humanitarian assistance inside South Sudan, including more than 1.9 million internally displaced people (IDPs).(UNHCR, 2017).

This review critically reviewed the article 'Public health activities in humanitarian emergencies case of South Sudan 'in various published and unpublished paper regarding Public Health humanitarian emergencies in South Sudan. The review first summaries the article. Secondly, briefly analyses the effectiveness of the article's structure, investigating how the information is set out and whether the reader can access it efficiently. Thirdly, the reviewed critique the article, evaluating its authority, currency, accuracy, objectivity and coverage. Reviewed the key result of studies and analyses the graph before finally judging the article's accessibility and credibility. Overall the article was well written, clear and relevant.

A country-wide economic crisis is deepening in South Sudan. With an inflation rate of 700 percent, most families cannot purchase their basic WASH supplies like safe water, soap and hygiene items. As a result the population has even less access to safe water and sanitation services than before the conflict. 
DOI: $10.21522 / \mathrm{TIJPH} .2013 .05 .04 . A r t 027$

ISSN: $2520-3134$

In addition to conflict and recurrent disease outbreaks, a range of other risks hamper access to WASH services in South Sudan. Flooding and drought have been reported in different locations. Both have a major impact on the WASH sector capacity. Flooding is common, particularly in areas with low altitude. Recently, $\mathrm{NBeG}$ and Jonglei states have been affected by flooding. Around 60 per cent of roads are impassable during the rainy season. Flooding is also a major contributor to communicable and water -borne diseases such as cholera, diarrhea, and malaria.

The continued insecurity, disease outbreaks, nutrition crises and other natural emergencies combined with poor infrastructure throughout the country highlight the need for UNICEF and its partners to continue providing emergency services to vulnerable communities. UNICEF is the Cluster lead for WASH and manages 75 per cent of the core pipeline supplies on behalf of the cluster partners for 2017.

The humanitarian crisis in South Sudan has deepened and spread as a result of multiple and interlocking threats, ranging from armed conflict and inter-communal violence, economic decline, disease, and climatic shocks. Hunger and malnutrition have reached historic levels. At the height of the lean season in July 2016, some 4.8 million people - more than one in three people in South Sudan - were estimated to be severely food insecure. This number is expected to rise as high as 5 million in 2017. The food security situation is at the most comprised level since the crisis commenced in 2013- the combination of conflict, economic crises, and lack of adequate levels of agricultural production combined have eroded vulnerable households ability to cope. More than one million children under age 5 are estimated to be acutely malnourished, including more than 273,600 who are severely malnourished. (UNOCHA, 2017).

Susceptibility to disease has risen after three years of conflict and crisis. More than 2 million cases of malaria were reported from January to November 2016; an increase compared to the same period in 2015. The cholera outbreak in 2016 caused more cases and spread to more locations than 2015 . There are rising cases of the deadly tropical disease kala-azar and more than twice the number of counties have been affected by measles outbreaks in 2016 (13) compared to 2015 (5). Violence and displacement in the Greater Equatoria region have affected populations with the highest prevalence rates of HIV/AIDS in South Sudan, cutting many off from life-sustaining treatment. (UNOCHA, 2016).

More than 125 million people across the globe require humanitarian assistance due to a combination of conflict-related crises and natural disasters, according to U.N. statistics. Some 60 million are displaced people and refugees. (Simmons, 2016).

The 'new humanitarianism', based on a rights based approach, argues that giving aid is more than just about saving life - it is also about tackling the root causes of conflict and delivering aid in a way that can ultimately reduce violence and promote recovery, development and peace. That might be credible for the case South Sudan IDPs, but the refugees, addressing the root cause of the problem and conflict might not be easy for the humanitarian community due new refugee's status and for them been far from their country. Thus, for the modern emergency health workers technical training by itself is not enough. They are also supposed to have a good understanding of International Humanitarian Law and Protection, be able to carry out context analysis and make linkages between short term relief and longer-term rehabilitation, recovery and peace-building. (Mitchell, 2003).

Evidence from Humanitarian emergencies studies confirm that majority of deaths in conflict settings are due to preventable communicable diseases and malnutrition, not violence. However there is no significant evidence from this very South Sudan Humanitarian emergency so far regarding death due to preventable communicable diseases and malnutrition verses death due to violence. Health service provision makes an essential contribution to the reduction of avoidable morbidity and mortality resulting from a crisis, and alleviates the suffering of people by providing curative and preventive care. Nutritional interventions are crucial in preventing and treating malnutrition, and complement livelihoods support, food aid and health care provision. Together, health and nutrition form an important sector in humanitarian aid. (UNHCR, 2017).

The Sphere Standards were created to help humanitarian aid workers understand and plan for how to best assist victims of disasters and other humanitarian emergencies. It is important to be familiar with these 
international minimum standards with regards to Water, Sanitation and Hygiene, Food, Shelter and Health indicators. One must also take into consideration issues of mental health and the particular challenges that arise with vulnerable populations.

UNHCR and partners joint statement on scaling up the community based health workforce for emergencies in Oct 2011 revealed the critical contribution of community health workers (CHWs) for all phase of emergencies management (prevention, preparedness, response and recovery, UNHCR south Sudan operation initiated The community based health programme (CBHP) should be used as strategy to improve access to basic and essential health service. The CBHP is a defined package that provides for essential promotion and preventive health services targeting households. It is designed to improve the health status of families, with their full participation, using local technologies and the community's skill and wisdom The CBHP, when properly employed, can significantly improve community access to essential and preventive health services and reduce maternal and child death in non-emergency and emergency setting. (UNHCR, 2017).

The most undeniable limitation was the fighting that broke out in Juba on 15 December 2013 marked the beginning of a violent civil war. At the time, South Sudan already had some of the worst development indicators globally, and NGOs were playing an important role in the delivery of basic services. The conflict took resident international NGOs - many of which were focused on development activities - by surprise. Expatriate staff were out of the country for the holiday, and within hours most of the others had evacuated. National NGOs were badly affected; offices were looted, South Sudanese aid workers were executed, vehicles were taken and borehole machines broken. National NGO staff, feeling aban Drive UNHCR vehicles for the transport of authorized passengers and delivery and collection doned by their international counterparts, describe how they 'woke up one day and everything had changed'. (HPN, 2017).

Despite multi-million dollar international interventions in this sector and the obvious importance of health in complex emergencies, there is surprisingly little common understanding among the organizations that fund and implement these interventions about the most appropriate and sustainable responses to the health needs and vulnerabilities of affected populations at various stages of complex emergencies. Nor is there a sufficient base of knowledge about how international interventions relate to and support local or indigenous initiatives and capacities in this sector. (WHO, 1989).

\section{Context of south sudan}

The January 2005 signing of the Comprehensive Peace Agreement (CPA) between the GoS and the southern based Sudan People's Liberation Army (SPLA) officially ended more than two decades of northsouth conflict during which famine, fighting, and disease killed an estimated 2 million people and displaced at least 4.5 million others within Sudan.

The GoRSS declared independence on July 9, 2011, after a referendum on self-determination stipulated in the CPA. Upon independence, USAID designated a new mission in Juba.

On December 15, 2013, clashes erupted in Juba between factions within the GoRSS and quickly spread into a protracted national conflict with Jonglei, Unity, and Upper Nile representing the primary areas of fighting and displacement. On December 20, 2013, USAID activated a Disaster Assistance Response Team (DART) to lead the USG response to the developing crisis in South Sudan. USAID also stood up a Washington, D.C.-based Response Management Team (RMT) to support the DART.

On August 26, 2015, GoRSS President Salva Kiir signed a peace agreement that the Sudan People's Liberation Army-In Opposition (SPLA-IO) and other stakeholders had signed on August 17. Opposition leader Riek Machar returned to Juba and was sworn in as the First Vice President (FVP) on April 26, 2016; GoRSS President Salva Kiir appointed a Transitional Government of National Unity on April 28.

Fighting between SPLA and SPLA-IO forces broke out in Juba on July 7, 2016, displacing thousands of people and prompting FVP Machar to flee. As a result, the U.S. Embassy in Juba ordered the departure of nonemergency USG personnel from South Sudan on July 10. Ongoing heightened tensions persist in the 
DOI: $10.21522 / \mathrm{TIJPH} .2013 .05 .04 . A r t 027$

ISSN: $2520-3134$

country, and the humanitarian situation remains precarious. On January 5, the U.S. Department of State ended the ordered departure status for the U.S. Embassy in Juba.

Insecurity, landmines, and limited transportation and communication infrastructure restrict humanitarian activities across South Sudan, hindering the delivery of critical assistance to populations in need.

On October 14, 2016, U.S. Ambassador Mary Catherine Phee redeclared a disaster in South Sudan for FY 2017 due to the humanitarian crisis caused by ongoing violent conflict, resultant displacement, restricted humanitarian access, and the disruption of trade, markets, and cultivation activities, which have significantly increased food insecurity and humanitarian needs.

Refugee: South Sudan hosted 261,500 refugees as of November 2016, and it is estimated that by the end of 2017 the refugee population will rise to 302,809 due to new arrivals, projected to be around 15,000, and natural growth. Refugees continue to arrive in South Sudan due to on-going fighting in South Kordofan and Blue Nile states in Sudan, while refugees from the Central African Republic, Democratic Republic of Congo and Ethiopia are in protracted displacement, mostly in Central and Western Equatoria. Over the course of 2016, the protective environment for refugees inside South Sudan deteriorated. The multiplicity of armed elements has exacerbated the challenge of maintaining the civilian character of asylum and inhibited humanitarians' access to refugee locations, particularly in Greater Equatorial. Filippo Grandi remarks at the opening of the High-level meeting to address large movements of refugees and migrants 19 September 2016, it is important that we talk about both refugees and migrants. There are many common features that we must join forces in tackling. But refugees have - and must retain - a distinct status, because conflict and persecution mean they cannot go home.

And with more than 65 million people forcibly displaced globally - as refugees or within their own countries - it's time to recognize that we cannot go on as before.

Evaluation of the health and nutrition sector is complicated, as there are many elements to be examined, such as the presence and capability of trained staff, drug supply systems, financing and health and nutrition information systems. Moreover, a health and nutrition response consists of a variety of interventions, ranging from direct life-saving interventions to comprehensive nutrition and health services (such as reproductive and mental health services). Services are provided in dynamic situations with fluctuating insecurity and population movements. Outcomes are difficult and expensive to measure, and it can be problematic to attribute outcomes to health and nutrition activities.

Health: (UNHOCHA, 2017) reports indicated that, after three years of conflict, the population is highly susceptible to disease, and more than 5 million people are in need of humanitarian healthcare services. Most health facilities are not functioning and those that are provide minimal services due to drug and staff shortages. Communicable diseases have spread in 2016, including cholera which has reached new locations along the River Nile, and there is a growing number of war wounded. Some 302,800 refugees will also require health assistance in 2017.

Despite the negative impacts of the ongoing conflicts on the overall health services provision, UNHCR and its partners managed to maintain uninterrupted provision of primary health care services in all camps.

A mixed methods assessment of barriers to maternal, newborn and child health in Gogrial West, South Sudan study done by (Lawry et. al, 2017) highlighted that Barriers to care for mothers, infants and children are far more than the lack of antenatal care. Maternal, newborn and child health suffers from lack of skilled providers, resources, distance to clinics. A lack of gender equity and accepted negative social norms impedes healthy behaviors among women and children.

\section{Nutrition}

Six million people in South Sudan face life-threatening hunger while more than four million people have been displaced from their homes, including two million refugees.

The nutrition crisis in South Sudan continues to escalate. More than one million children under age 5 and over 339,000 pregnant and lactating women are estimated to be acutely malnourished and in need of life-saving nutrition services. In 2016, 32 out of 44 of the SMART surveys conducted reported global acute 
malnutrition (GAM) levels above the emergency threshold of 15 per cent. Of these, 13 found a GAM prevalence by Weight-for Height of more than 25 per cent. The GAM rate was above the catastrophe threshold in Gogrial West in Warrap and Renk in Upper Nile (>30 per cent), and just beneath it in Abiemnhom and Rubkona in Unity (29.2 per cent GAM). Pregnant and lactating women (PLW) have increased nutritional requirements and, if not supported, can become malnourished, potentially leading to miscarriages, pre-mature deliveries and low birth weight. The elderly also face heightened vulnerability. Some 302,800 refugees are also expected to need nutritional assistance in 2016.

\section{FAO south sudan situation report - February 2017}

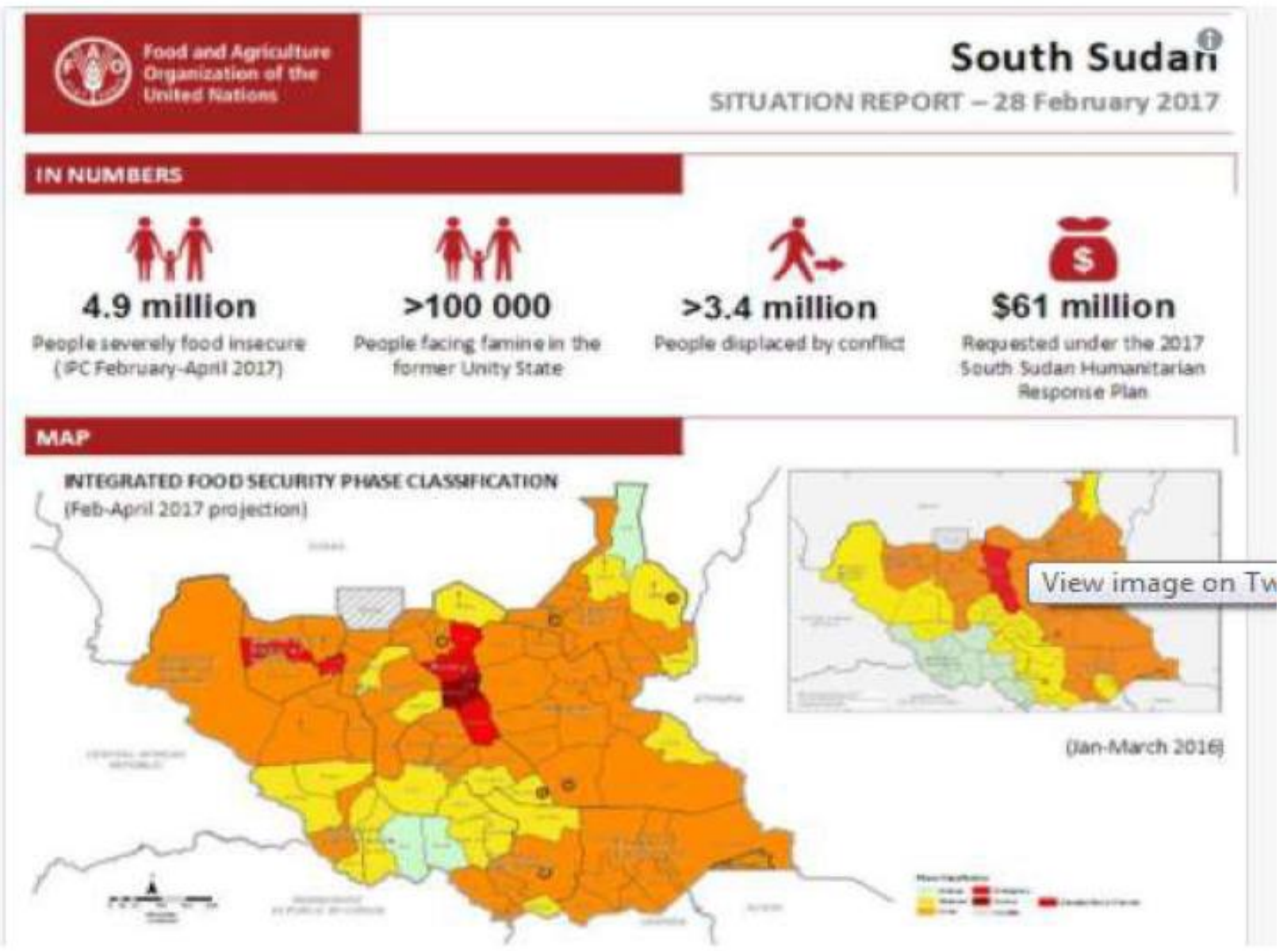

Water and Sanitation: Nearly 4.8 million of the most vulnerable people across South Sudan are in need of support to access safe water and basic sanitation facilities. It is estimated that only 41 per cent of the population have access to safe water 7 whereas about 74 per cent of the total population defecate in open. As the conflict spread to new areas in 2016, boreholes were damaged or made dysfunctional due to lack of repairs. A cholera epidemic was again declared in 2016. Access to safe water in urban areas has reduced as a result of rapidly rising prices. Some 302,800 refugees will also require WASH services in 2017. 
DOI: $10.21522 / \mathrm{TIJPH} .2013 .05 .04 . A r t 027$

ISSN: $2520-3134$

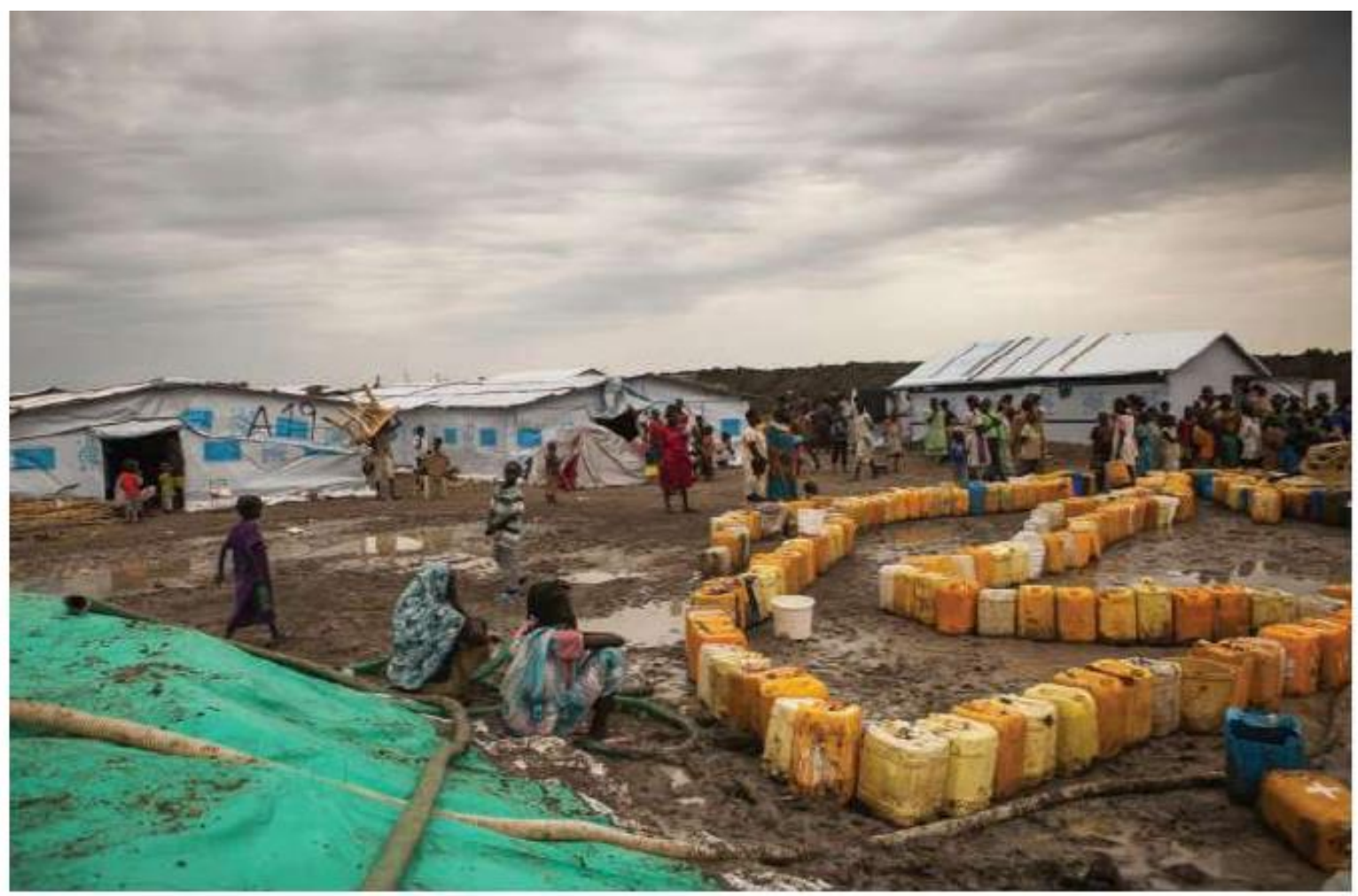

Women and children seek protection in a UN Protection of Civilians site 2015 (HPN, 2017).

A country-wide economic crisis is deepening in South Sudan. With an inflation rate of 700 percent, most families cannot purchase their basic WASH supplies like safe water, soap and hygiene items. As a result the population has even less access to safe water and sanitation services than before the conflict.

In addition to conflict and recurrent disease outbreaks, a range of other risks hamper access to WASH services in South Sudan. Flooding and drought have been reported in different locations. Both have a major impact on the WASH sector capacity. Flooding is common, particularly in areas with low altitude. Recently, $\mathrm{NBeG}$ and Jonglei states have been affected by flooding. Around 60 per cent of roads are impassable during the rainy season. Flooding is also a major contributor to communicable and water -borne diseases such as cholera, diarrhea, and malaria.

The continued insecurity, disease outbreaks, nutrition crises and other natural emergencies combined with poor infrastructure throughout the country highlight the need for UNICEF and its partners to continue providing emergency services to vulnerable communities. UNICEF is the Cluster lead for WASH and manages 75 per cent of the core pipeline supplies on behalf of the cluster partners for 2017.

\section{Gabs in provision of the assistance globally}

Humanitarian access in South Sudan remains a challenge, largely due to poor infrastructure and an overreliance on air transport, there are dead end all around, south Sudan is unmatched anywhere in the world, an unpredictable and volatile security environment, and the move away from static and broad frontlines to more fluid and micro frontlines.

Besides being the largest refugee crisis in Africa ... South Sudan is one of the greatest tragedies of our time," UN High Commissioner for Refugees Filippo Grandi told a donor conference in Geneva at which the appeal was launched on Monday (UNHCR, 2017).

South Sudan is a country that is affected by complex emergencies resulting from prolonged conflict, climate change, a broken health system and outbreaks of communicable diseases.

This situation is further exacerbated by the international community's lukewarm response to Africa's refugee problems. For example, in 1999 , the international community spent $\$ 0.11$ per refugee, per day in 
Africa. In contrast, it spent $\$ 1.28$ per day per person in the Balkans. Furthermore, the international community spent $\$ 10$ million a week on Kosovar refugees in Albania and Macedonia. Yet, in the same year, UNHCR's annual appeal for \$8 million for refugees in West Africa only raised \$1.3 million. (Lomo, 2000).

Health standards in protected villages and camps for IDPs are far from ideal. Water in camps is scarce. The environment as a whole in these camps is appalling and the population is subjected to frequent epidemics of cholera and other enteric diseases. There are generally not enough dispensaries and those that do exist have neither sufficient resources, nor qualified staff to meet the needs of the internally displaced people. Although it is generally accepted that refugees enjoy higher standards of health services than do the locals, these services depend on unreliable international funding. Moreover, many refugees would argue that these "higher" standards do not necessarily mean services are always "adequate." 23 The major problem faced by both refugees and internally displaced persons, in settlements and camps respectively, is accessing enough land to feed themselves, a fact that further illustrates how the enjoyment of the right to health is contingent on the freedom of movement.(Lomo, 2000)

\section{Human resources for health challenges in fragile country}

South Sudan humanitarian crises has resulted in a general collapse of formerly existing health program, with no quick-fix solution easily identified. Greater financial and human resources are needed to fill gaps, Emigration of health professionals from fragile and conflict-affected states FCAS can create a negative feedback loop for health outcomes and highlights the important challenges surrounding sustainable human resources for health (HRH) in fragile states.( MacKinnon \& MacLaren, 2012)

In humanitarian setting the health service and nutrition and food aid are delivered as part of the measures of humanitarian assistance. The Public health services have shown enhancements subsequently from the time when the humanitarian assistance was initiated. Though, Public health service delivery still faces several challenges: ranging from insufficient health systems in line for the limited and poor health facilities/infrastructure (still in temporary facilities in tents/rub halls), limited health human resources. (UNHCR, 2016), Absence of healthy behaviour and high use of traditional healers and practices by the community. Scanty health service utilization, particularly the maternal and child health service and EPI. Disproportionately high burden of communicable diseases such as RTI, shigellosis, acute watery diarrheal diseases, malaria etc. The risk of outbreaks are very high in camps include cholera, meningitis, measles, bloody and acute water diarrheal diseases.

TB should not be ignored in chronic complex emergencies. With flexible and adapted programmes and investment in appropriate communication technology, acceptable treatment outcomes can be obtained. Useful adaptations include provision of accommodation near to treatment as well as emergency drug packs in case of increased instability. Remote management of programmes is feasible with sufficient resources and motivated and well supported local staff.

\section{The future of humanitarian action in south sudan}

It's not a humanitarian agencies that's going to solve the Public health humanitarian emergencies in South Sudan, than the political will and support.

\section{Learning and competency in humanitarian operations}

Historically humanitarian agencies have emphasized 'technical' knowledge and skill, complemented by management administration skills (especially financial management and planning, proposal and report writing). Evidence from (Mitchell, 2003) study suggested that there is greater need to understand the context in which one operates, not just in terms of the institutional environment (with its policies, budgets and institutional dynamics), but also in its historical, socio-cultural and political dimensions. And that sound very important as most of the humanitarian agencies lunch their operation where there is no one to be referred to as institutional mummery guru on the ground, Additionally skill in relationship management is now recognized as a vital requirement for 'good performance'. Hence the interest in 'leadership' and 
DOI: $10.21522 / \mathrm{TIJPH} .2013 .05 .04 . A r t 027$

ISSN: $2520-3134$

'leadership training', a rather fuzzy label that can cover different ingredients and that begs the question whether you can develop personality traits through 'training'

Minear3 identifies four impediments to learning: the tendency of humanitarian organizations to approach every crisis as unique, their action-oriented nature, their defensiveness to criticism, and their lack of accountability

\section{Methodology}

The study was based on desk research and a review of the secondary literature on Public health activities in humanitarian emergencies, the principle investigator first summaries the articles from South Sudan NGOs forum, Web of Science (2005- 2017), Proust (2004- 2017) and PubMed (2005-2017). The years of publication were restricted to the last decade which ensures that the data is current and credible. Public health measures included the mortality, morbidity rates and Malnutrition rate amongst the refugees and internally displaced persons. Secondary references were then gathered from the primary sources. The abstracts were selected randomly and independently

Principal investigator set out the information's in such way that it's efficiently accessible. The authors drew heavily from the World Health Organization's, UNHOCHA and UNHCR operations reports and donor-funded health assessments and surveys by the South Sudan ministry of health.

Study population: South Sudan gained independence on July 9th, 2011. It is divided into three regions, Bar el Ghazal, Equatorial, and Greater Upper Nile and thirty two states within those regions. The population is approximately 11 million people, predominately living in rural subsistence farming communities. South Sudan has the third largest oil reserves in Sub-Saharan Africa and is rich with other natural resources like copper, iron ore, and arable land for agriculture.

Only $27 \%$ of the population can read and write in the national language, English.

\section{Results}

The humanitarian crisis in South Sudan has deepened and spread as a result of multiple and interlocking threats, ranging from armed conflict and inter-communal violence, economic decline, disease, and climatic shocks. Hunger and malnutrition have reached historic levels. At the height of the lean season in July 2016, some 4.8 million people - more than one in three people in South Sudan - were estimated to be severely food insecure.

The complexity and the unparalleled dynamic of South Sudan conflict increases the population susceptible to disease, Six million people in South Sudan face life-threatening hunger while more than four million people have been displaced from their homes, including two million refugees.

Given the ongoing conflict in country and the limited capacity government line ministries, the possibility of handing over all Public health activates to the government/Counties authorities where persons of concern to Humanitarian agencies are settle seem bleak. The need for a longer term transition plan/strategy and engaging with development oriented partners is critical.

Evidence across the globe of persons of concern to Humanitarian agencies shows that, access of primary healthcare amongst the persons of concern to Humanitarian agencies is overwhelmingly shaped by the regulations of the migration process and legal frameworks of individual states and nations' the persons of concern to Humanitarian agencies should not get services same as host community, but the case of South Sudan the host community them same selves are at same catastrophe as IDPs or refugees.

South Sudan is not getting enough assistance and appropriate international community focused, The U.N. appealed for $\$ 1.6$ billion to assist 4.6 million people in need in 2015, but the effort was only 62 percent funded. Only 88 percent of the $\$ 1.29$ billion requested for 2016 has been funded. It's not a humanitarian agencies that's going to solve the Public health humanitarian emergencies in South Sudan, than the political will and support.

The conflict led to a "paralyzing funding shortfall" also led to the suspension and closer of humanitarian agencies operation and projects, in many places around South Sudan, that's mean health clinics were close, 
and more live saving project were short down across parts of South Sudan leaving some people without access to healthcare, according to the agency.

South Sudan faces some of the steepest healthcare challenges globally as most of the population is affected by a persistent health crisis. Facilities are run by under resourced paraprofessionals with limited capacities due to shortages of trained health professionals. Furthermore, healthcare facilities have limited support and resources for infrastructure rehabilitation and repair.

Indications reviled that, there is a severe shortage of health care services and professionals (only one doctor for every 100,000 people). Medical facilities are under-equipped and unhygienic. Since many South Sudanese do not have access to clean water, deadly diseases such as malaria continue to spread.

Evidence from Humanitarian emergencies studies confirm that majority of deaths in conflict settings are due to preventable communicable diseases and malnutrition, not violence. However there is no significant evidence from this very South Sudan Humanitarian emergency so far regarding death due to preventable communicable diseases and malnutrition verses death due to violence.

Women and girls, in particular, are affected by the crisis, many facing violence, abuse and exploitation daily. Thousands lack the care they need to cope with unwanted pregnancies and pregnancy complications.

Susceptibility to disease has risen, with repeated outbreaks of vaccine preventable diseases, including measles, in the country. Water-borne and hygiene related diseases such as watery and bloody diarrhea, and Hepatitis are still common, after three years of conflict and crisis, the burden is high due to: the environment as a whole in these camps is appalling and the population is subjected to frequent epidemics of cholera and other enteric diseases. There are generally not enough dispensaries and those that do exist have neither sufficient resources, nor qualified staff to meet the needs of the internally displaced people. Although it is generally accepted that refugees enjoy higher standards of health services than do the locals, these services depend on unreliable international funding. Moreover, many refugees would argue that these "higher" standards do not necessarily mean services are always "adequate."

The authority Pipeline breaks: medical items; long delays in receiving tax exemption letters; and at times delays in procurement.

Refugee and most of IDPs is currently having $100 \%$ access to ARTs, enough focused on hygene promotion have been accelerate compare to the beginning of emergencies

\section{Discussion}

South Sudan is not getting enough assistance and appropriate international community focused, this could be due to many factors as indicated by ( Simmons, 2016) regarding cash for crises, The reason some countries attract more funding varies, include factors such as the scale of the tragedy, media attention given to a certain crisis, and geographical and historical ties. Donors are pulled in different directions by multiple large-scale crises that are happening at the moment and are faced with tough decisions about where to allocate their finite resources.

The humanitarian crisis in South Sudan has deepened and spread as a result of multiple and interlocking threats, ranging from armed conflict and inter-communal violence, economic decline, disease, and climatic shocks, That's shows that it's not a humanitarian agencies that is going to solve South Sudan Crises rather the political support and it's not going to happen soon, their more strategic measure is need to assist persons of concern to humanitarian community.

The developmental institution in South Sudan was not design to respond to over burden situation after independence of South Sudan leave alone the humanitarian emergencies and crises, that's why Susceptibility to disease has risen after three years of conflict and crisis and deterioration of health status of community was well observed.

Substantiation from South Sudan Humanitarian operations shows that, evaluations of activities in the health and nutrition sector tend to be limited to single-agency project evaluations. Historically there has been a lack of sector-wide evaluations even though only sector-wide evaluations can examine issues such as coverage and the appropriateness of the choices of, and sharing of the lessons learn from the effective 
DOI: $10.21522 / \mathrm{TIJPH} .2013 .05 .04 . A r t 027$

ISSN: $2520-3134$

interventions. Therefore sector-wide evaluations should be coordinated by lead agency in particular operation to measure the impact and the outcomes.

The common way utilized by hygiene promotion implementers to measure performance is through Knowledge Attitude and Practice Surveys (KAP) in which baseline knowledge, attitude and practice levels are measured at the beginning of a response and then using this baseline to compare changes in knowledge, attitudes and behaviour at or near the end of the intervention from an end line KAP survey. The problem with KAP surveys is that they are prone to social desirability bias (the respondent gives the answer they think the enumerator wants to hear).

Proof indicate that, it's not a humanitarian agencies that's going to solve the Public health humanitarian emergencies in South Sudan, than the political will and support. The world must put effort to end the conflict where by the humanitarian emergences will come to an end.

\section{Conclusion}

The study sought to analyze the dynamics involved in Public health activities in humanitarian emergencies and the analysis revealed that:

South Sudan situation cannot be put in to one box, because it has many vigorous, therefore need different approaches and interventions. The study recommends that improved data are imperative towards supporting inter-sectorial work in addressing Public health care needs of the persons of concern to Humanitarian agencies.

Given the ongoing conflict in country and the limited capacity government line ministries, the possibility of handing over all Public health activates to the government/Counties authorities where persons of concern to Humanitarian agencies are settle seem bleak and unwelcoming. The need for a longer stretch transition plan/strategy and appealing with development oriented partners is precarious.

The complexity and the unparalleled dynamic of South Sudan conflict increases the population susceptible to disease, Six million people in South Sudan face life-threatening hunger while more than four million people have been displaced from their homes, including two million refugees, the is need to improve the early crises and emergency warning system in South Sudan to promptly detect and responded to the emergencies.

Long-lasting scarcity and high turn-over of qualified staff remains a challenge. It's important to support/invest in local capacity building for qualified refugees/IDPs and local host communities through enrollment in formal educational institution and it's highly important to continually build the capacity of community health workers and preposition essential drugs and medicals supplies to ensure continuity of basic services at times of security crisis.

Confirmation from South Sudan Humanitarian operations shows that, evaluations of activities in the health and nutrition sector tend to be limited to single-agency project evaluations. Historically there has been a lack of sector-wide evaluations even though only sector-wide evaluations can examine issues such as coverage and the appropriateness of the choices of, and sharing of the lessons learn from the effective interventions. Therefore sector-wide evaluations should be coordinated by lead agency in particular operation to measure the impact and the outcomes.

Proof indicate that, it's not a humanitarian agencies that's going to solve the Public health humanitarian emergencies in South Sudan, than the political will and support. The world must put effort to end the conflict where by the humanitarian emergences will come to an end.

\section{Future steps}

Studying and analyzing the dynamics involved in Public health activities in humanitarian emergencies and the equipped principal investigator with broad knowledge of the dynamic of the Public health aspect of South Sudan Humanitarian emergency and there for identify the research gabs in the area as follow:

There is need for father detailed research and study, to evaluate protection aspect to gain an understanding of the political context of the crisis, including who gains and losses from the conflict, and how this impacts on Public health activate delivery in Humanitarian setting. 
It's prerequisite to study technical and non-technical challenges surrounding Health informatics records in Public health during humanitarian emergencies in South Sudan.

\section{References}

[1]. Ann M. Simmons, 2016, Cash for crises: Who gives, who gets and why it's not enough, http://www.latimes.com/world/global-development/la-fg-global-humanitarian-funding-snap-story.html.

[2]. Humanitarian Practice Network, 2017, the crisis in South Sudan number 68, January 2017 http://odihpn.org/wpcontent/uploads/2017/01/HE-68-web.pdf.

[3]. (HPN) Humanitarian Practice Network, 2017, Humanitarian Exchange, Special feature the crisis in South Sudan.

[4]. Interagency Health and Nutrition Evaluations in Humanitarian Crises (IHE) Initiative, 2007, Guidelines for Interagency Health and Nutrition Evaluations in Humanitarian Crises.

[5]. James MacKinnon and Barbara MacLaren, 2012, Human Resources for Health Challenges in Fragile States: Evidence from Sierra Leone, South Sudan and Zimbabwe.

[6]. John Mitchell, Coordinator, ALNAP Secretariat WHO, 2003, Health in Humanitarian Emergency. http://www.who.int/hac/about/9545.pdf.

[7]. Lawry et al. Reproductive Health (2017), A mixed methods assessment of barriers to maternal, newborn and child health in Gogrial West, South Sudan 14:12 DOI 10.1186/s12978-016-0269-y.

[8]. Special Feature: Comprehensive CME Program on Disaster Medicine Part 1 Conferences and Lectures, International Standards for Public Health Activities JMAJ 56(1): 15-18, 2013 https://www.med.or.jp/english/journal/pdf/2013_01/015_018.pdf.

[9]. UNHCR, 2016, Community Based Health, Nutrition and Hygiene Promotion Program in Refugees Setting, Maban, South Sudan.

[10]. UNHCR, 2017 Guidelines for Interagency Health and Nutrition Evaluations in Humanitarian Crises, 2007, Interagency Health and Nutrition Evaluations in Humanitarian Crises (IHE) Initiative, p 1.

[11]. UNHCR, 2017, As South Sudan crisis deepens, more funds needed for refugees http://www.unhcr.org/news/latest/2017/5/5919a18f4/south-sudan-crisis-deepens-funds-needed-refugees.html.

[12]. UNHCR, 2017, SOUTH SUDAN SITUATION 2017 http://www.unhcr.org/partners/donors/593e9e9b7/unhcrsouth-sudan-revised-supplementary-appeal-january-december-2017.html?query=South\%20Sudan\%20Crises.

[13]. WHO, 1998, Health Interventions in Complex Emergencies a Case Study of Liberia http://www.who.int/hac/techguidance/training/analysing_health_systems/liberia_health_interventions_complex_eme rgencies.pdf.

[14]. WHO. Health Action in Crisis - definitions. Available from: http://www.who.int/hac/about/definitions/en/.

[15].Zachary A. Lomo, the Struggle for Protection of the Rights of Refugees and IDPs in Africa: Making the Existing International Legal Regime Work, 18 Berkeley J. Int'l Law. 268 (2000). Available at: http://scholarship.law.berkeley.edu/bjil/vol18/iss2/8. 